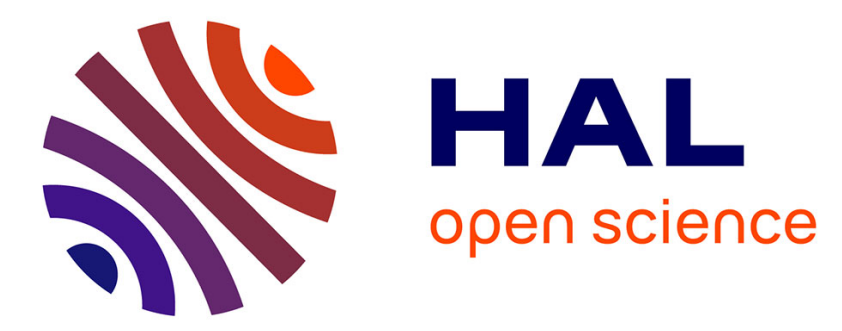

\title{
Microwave-ultrasound simultaneous irradiation: a hybrid technology applied to ring closing metathesis
}

Martina Sacco, Clarence Charnay, Francesco de Angelis, Marilena Radoiu, Frédéric Lamaty, Jean Martinez, Evelina Colacino

\section{- To cite this version:}

Martina Sacco, Clarence Charnay, Francesco de Angelis, Marilena Radoiu, Frédéric Lamaty, et al.. Microwave-ultrasound simultaneous irradiation: a hybrid technology applied to ring closing metathesis. RSC Advances, 2015, 5 (22), pp.16878-16885. 10.1039/c4ra14938f . hal-01114826

\author{
HAL Id: hal-01114826 \\ https://hal.science/hal-01114826
}

Submitted on 5 Mar 2021

HAL is a multi-disciplinary open access archive for the deposit and dissemination of scientific research documents, whether they are published or not. The documents may come from teaching and research institutions in France or abroad, or from public or private research centers.
L'archive ouverte pluridisciplinaire HAL, est destinée au dépôt et à la diffusion de documents scientifiques de niveau recherche, publiés ou non, émanant des établissements d'enseignement et de recherche français ou étrangers, des laboratoires publics ou privés. 


\title{
Microwave-ultrasound simultaneous irradiation: a hybrid technology applied to ring closing metathesis $\uparrow$
}

\author{
M. Sacco, ${ }^{\text {ab }}$ C. Charnay, ${ }^{\star c}$ F. De Angelis, ${ }^{b}$ M. Radoiu, ${ }^{d}$ F. Lamaty, ${ }^{a}{ }^{2}$. Martinez ${ }^{a}$ \\ and E. Colacino*a
}

\begin{abstract}
A new hybrid microwave (MW) and ultrasound (US)-assisted reactor concept was investigated. The 2.45 $\mathrm{GHz}$ microwaves were delivered by a semiconductor generator via a coaxial cable to a monomode Transverse Electric (TE) microwave resonant cavity within which the reactor was placed; the US (25 kHz) were delivered at the bottom of the TE cavity via a novel designed sonotrode consisting of a detachable metallic plate-probe (indirect sonication). The semiconductor microwave generator helped to optimize the absorbed energy via its automatic frequency tuning function. The dual MW/US device allowed the use of both technologies separately or in a simultaneous combined manner. The ring-closing metathesis of diethyl diallyl malonate in glycerol micellar conditions was studied as an example using this novel hybrid technology. The results were compared with those obtained when microwaves or ultrasound were applied individually. Various benzylidene-, indenylidene- and Hoveyda-Grubbs-type catalysts were screened. The novel reactor for combined MW/US irradiation showed beneficial effects on the outcome of the reaction.
\end{abstract}

\section{Introduction}

The development of environmental-friendly synthetic processes is an economical and societal concern and one of the major questions in academia and industry. The replacement of classical volatile organic solvents by new sustainable media, ${ }^{\mathbf{1 , 2}}$ coupled to energy saving ${ }^{3,4}$ and selective protocols for catalytic and organic processes is an attractive challenge to be taken up. Combining different sources of energy with their own specific effects constitutes a very interesting approach towards more reliable and cost-effective synthetic transformations. ${ }^{5-9}$ In the 1990s, description of the first apparatus allowing the simultaneous use of green activation techniques such as microwaves and ultrasound was reported, ${ }^{\mathbf{1 0}}$ as well as its seminal application in the field of organic synthesis. ${ }^{11,12}$ Either small reaction volumes, insufficient adapted accessories or the absence of

${ }^{a}$ Institut des Biomolécules Max Mousseron (IBMM), UMR 5247 CNRS - Université Montpellier - ENSCM, Green Chemistry and Enabling Technology Team, Université Montpellier, Place E. Bataillon, 34095 Montpellier Cedex 5, France. E-mail: evelina. colacino@univ-montp2.fr; Web: http://www.greenchem.univ-montp2.fr/

${ }^{b}$ Dipartimento di Scienze Fisiche e Chimiche Università dell'Aquila e Consorzio INCA, Via Vetoio, Coppito, 67100 L'Aquila, Italy

'Institut Charles Gerhardt Montpellier, UMR 5253 CNRS-UM2, CC1502, Université Montpellier, Place E. Bataillon, 34095 Montpellier Cedex 5, France. E-mail: clarence.charnay@univ-montp2.fr

${ }^{d}$ SAIREM, 12, porte du Grand Lyon, BP 80214, 01702 Neyron Cedex, France suitable equipments on the market limited the expansion of this hybrid technology, which gained interest recently, especially in the field of organic synthesis ${ }^{13-22}$ and palladiumcatalyzed reactions. ${ }^{23-25}$ At earlier stage, reactions in organic solvents (DMSO, DMF, DMA or MeOH), ${ }^{\mathbf{1 0 , 1 9 , 2 0 , 2 2 , 2 5}}$ water $^{\mathbf{1 5 - 1 7 , 2 1 , 2 4}}$ or neat conditions ${ }^{\mathbf{1 1 , 1 2 , 1 4 , 1 8}}$ were reported, but very scarce attention was devoted to the application of the simultaneous microwaves-ultrasound irradiation (SMUI) hybrid technology in glycerol media. ${ }^{23}$ The only example of the use of hybrid SMUI technology in glycerol as solvent described the rutheniummediated transfer hydrogenation and palladium-catalyzed Suzuki-Miyaura cross-coupling reactions, in a house-made multimodal microwave cavity equipped with a pyrex horn. ${ }^{23}$ However, SMUI was scarcely explored to promote organic transformations and comprehensive studies in glycerol are still missing. Glycerol is a very suitable solvent for both microwave or ultrasound promoted reactions: ${ }^{26}$ because of its polarity (dielectric constant $\varepsilon=42.5$ ) and thermal conductivity $(\kappa=$ $0.285 \mathrm{~W} \mathrm{~m}^{-1} \mathrm{~K}^{-1}$ at $\left.300 \mathrm{~K}\right)$ glycerol absorbs microwave irradiation strongly, while its negligible vapour pressure $(0.0025 \mathrm{~mm}$ $\mathrm{Hg}$ at $\left.50^{\circ} \mathrm{C}\right)$ and viscosity $\left(\eta=1.069 \mathrm{~Pa} \mathrm{~s}\right.$ at $\left.20^{\circ} \mathrm{C}\right)$ both make it suitable also as cavitational media, especially when high temperature is required (e.g. $100{ }^{\circ} \mathrm{C}$ ), not possible for ultrasound-promoted reaction in water. Despite its importance for many industrial and pharmaceutical preparations and its high potential application as an environmentally friendly solvent to perform green synthesis, the use of glycerol is still limited in organic transformation. This is mainly due to: (i) the 
intrinsic reactivity of the polyol backbone leading to the formation of side products; (ii) the very poor solubility of the vast majority of organic compounds and (iii) the high viscosity leading to poor mass transport (at room temperature or under mild heating).

The fascinating possibility of applying SMUI hybrid technology to micellar catalysis in glycerol opens novel frontiers to impulse its expansion in synthesis and processing, allowing at the same time to overcome all the drawbacks described so far on the use of glycerol as reaction solvent. This approach benefits at the same time of the effective heating of glycerol under microwave irradiation, the efficient agitation provided by ultrasound and avoids side-reactions involving glycerol, the reactants being confined into the hydrophobic environment inside the micelle nanoreactor, allowing both the better diffusion of organic substrates into the glycerol phase and inhibition of its reactivity. ${ }^{27,28}$

This study is divided into two sections. The first part is devoted to the description of a novel prototype of monomodal apparatus, developed in our laboratories, able to provide simultaneous microwave-ultrasound irradiation (SMUI). The second part of the research illustrates the efficiency of the SMUI applied to one of the most powerful reaction for the formation of carbon-carbon double bonds, olefin metathesis. ${ }^{29}$ The reaction is usually performed in chlorinated or aromatic solvents, however, in view of the REACH (Registration, Evaluation, Authorisation and Restriction of Chemicals) legislation ${ }^{30}$ the use of these chemicals, posing adverse health and safety issues or demonstrates a detrimental environmental impact, will be phased out, affecting industries throughout the world. Isolated reports described olefin metathesis performed in environmentally friendly benign reaction media [e.g. water, ${ }^{31}$ methyl tert-butyl methyl ether (MTBE), ${ }^{32}$ dimethylcarbonate (DMC), ${ }^{33,34}$ glycerol, $^{35}$ poly(ethylene) glycols (PEGs), ${ }^{36}$ 2-methyltetrahydrofuran (2-MeTHF), ${ }^{37}$ acetic acid $^{38}$ or supercritical- $\left.\mathrm{CO}_{2}\left(\mathrm{sc}-\mathrm{CO}_{2}\right)^{39-41}\right]$. Aqueous micellar conditions were also investigated, ${ }^{42}$ in the presence of specially designed catalysts.

With the aim of developing sustainable synthetic processes, using possible alternative substances and/or technologies, the attention was focused towards the ruthenium-catalyzed ring closing metathesis (RCM) of diethyl diallylmalonate (DEDAM) 1 in a non-conventional media such as glycerol micellar conditions $^{28}$ (Scheme 1).

It is well accepted that in protic (alcoholic) solvents ruthenium hydrides are formed, ${ }^{\mathbf{4 3}, 44}$ leading to isomerization byproducts during RCM. In this context, micellar conditions will allow to perform RCM also in a polyol solvent (glycerol), with the reaction taking place into the micelle core. In addition, the possibility to replace harmful chemicals with green solvents and to develop new technologies will enhance innovation and competitiveness. Since MW and US allowed different irradiation mechanisms leading to different molecular driving forces, the combination of these two types of irradiation and its application in the RCM reaction is really appealing. We have previously described how the ruthenium-catalyzed ring closing metathesis (RCM) of diethyl diallylmalonate 1 under microwave activation in micellar condition ${ }^{28}$ was able to inhibit glycerol reactivity. ${ }^{35}$ The results presented herein aimed to extend the investigation

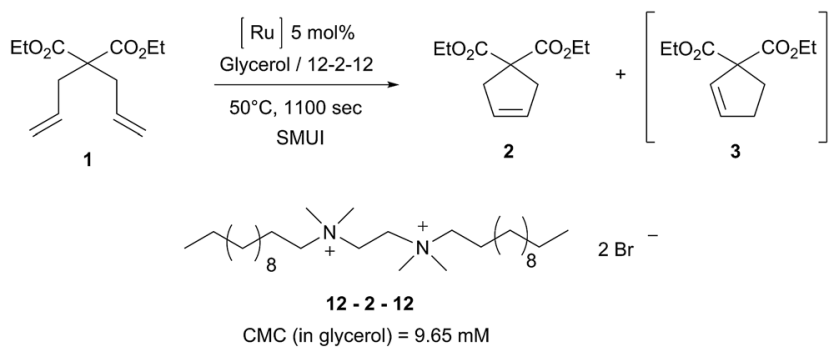

$[\mathrm{Ru}]:$
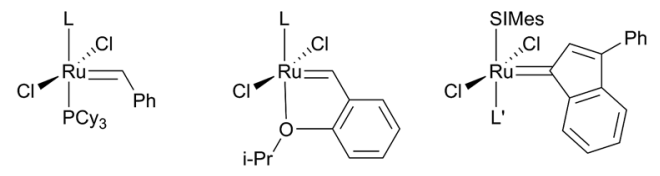

G

H-G

M2 $\mathrm{L}^{\prime}=\mathrm{PCy}_{3}$

G-I, HG-I $L=P C y_{3}$

G-II, HG-II, L = SIMes

$M 2_{0} L=P P h_{3}$

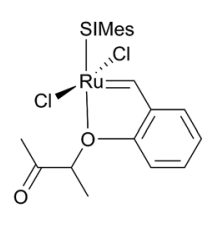

M5

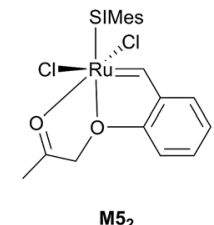

$\mathrm{M}_{2}$
Scheme 1 RCM in glycerol micellar conditions under SMUI.

of RCM reaction not only to a largest panel of commercially available catalysts, but also to evidence any synergetic effect coming from the combination of these two types of irradiation, electromagnetic $(2.45 \mathrm{GHz})$ and mechanical $(25 \mathrm{kHz})$. The results were also compared with those obtained when the reaction mixture was irradiated separately with either MW or US, and in some cases, using an oil bath.

\section{Results and discussion}

The use of non-traditional energetic sources like the simultaneous irradiation with MW and US, involves both technical and safety considerations. Some adaptations were already reported $^{\mathbf{6 , 4 5 , 4 6}}$ mainly for multimodal microwave reactors. Some flow ${ }^{47}$ or loop ${ }^{48}$ reactors equipped with ultrasound and microwaves generators were also described for scaling up. Technically, three general experimental methods were proposed to avoid short circuits, microwave leakage, sparks or explosions: (i) the source of ultrasound (a non-metallic horn) is immersed into the reaction liquid in the microwave cavity ${ }^{49}$ (direct sonication); (ii) the metallic ultrasound probe (usually titanium alloys) is not in direct contact with the reactive mixture but it is placed at the bottom of the reactor ${ }^{\mathbf{1 1}}$ (indirect sonication) and (iii) the microwave and ultrasound (titanium horn) are both immersed in the reacting liquid and delivered orthogonally ${ }^{50,51}$ (direct sonication). In these set-ups the ultrasound power delivered to the liquid depended on the type of material used for the horn. It was demonstrated ${ }^{52}$ that the strength of ultrasound irradiation was lower for pyrex than for a metallic horn; ceramics, quartz, ${ }^{53}$ or engineered plastics ${ }^{54}$ such as PEEK (polyether ether ketone) 
or PTFE (polytetrafluoroethylene), inert to microwaves, can also be used. In the field of MW/US organic synthesis applications, the direct sonication was mainly used due to the easiness of modifying a professional multimodal microwave oven to be equipped with a pyrex horn ${ }^{\mathbf{1 4 - 1 8 , 2 0 - 2 2 , 2 4 , 2 5}}$ inserted directly into the multimodal cavity. On the contrary, examples using indirect sonication remained scarce and were limited to esterification reactions ${ }^{11}$ or to the synthesis of nanowires, ${ }^{55}$ performing the experiment in a monomodal apparatus equipped with external metallic ultrasound probe. ${ }^{56}$ Until now, the main limitation to the development of indirect sonication in monomodal apparatus was probably the absence of dedicated equipments on the market. In this perspective, and to contribute to a faster development of SMUI technology, we developed a novel and efficient setup by adding a sonotrode to a professional monomode microwave reactor, Miniflow 200SS by Sairem ${ }^{57}$ (Fig. 1).

The emission of ultrasound waves $(25 \mathrm{kHz}, 200 \mathrm{~W})$ was made at the bottom of the reactor, via a novel designed, removable metallic plate-probe (sonotrode), which was not in direct contact with the reactive mixture and was placed far from the electromagnetic field (Fig. 1). Propagation of the ultrasound waves into the reactor was allowed by means of water standing in the bottom part of the U-shaped waveguide (see Fig. 1 in ESI $\dagger$ ) and outside the microwave irradiation zone, without interfering with the microwave absorption by the sample.

The simultaneous MW/US experiments were always realized by placing the pyrex reactor inside the MW cavity at the same height from the bottom of the sonotrode $(3.5 \mathrm{~cm})$ and simultaneously switching on both the microwave and ultrasound devices. The possibility of having real time linear measurements of both the reflected $\left(P_{\mathrm{r}}\right)$ and forwarded $\left(P_{\mathrm{f}}\right)$ microwave power levels allowed for precise calculation of the microwave power absorbed $\left(P_{\mathrm{a}}=P_{\mathrm{f}}-P_{\mathrm{r}}\right)$ by the reaction during the syntheses. In other words, the direct measurement of MW forwarded and reflected power values enabled the calculation of the energy $\left(E_{\mathrm{a}}\right)$ effectively absorbed by the irradiated sample $\left(E_{\mathrm{a}}=P_{\mathrm{a}} \times t\right.$, with $P_{\mathrm{a}}=$ absorbed power and $t=$ time, losses by convection are neglected). In addition, due to the use of a

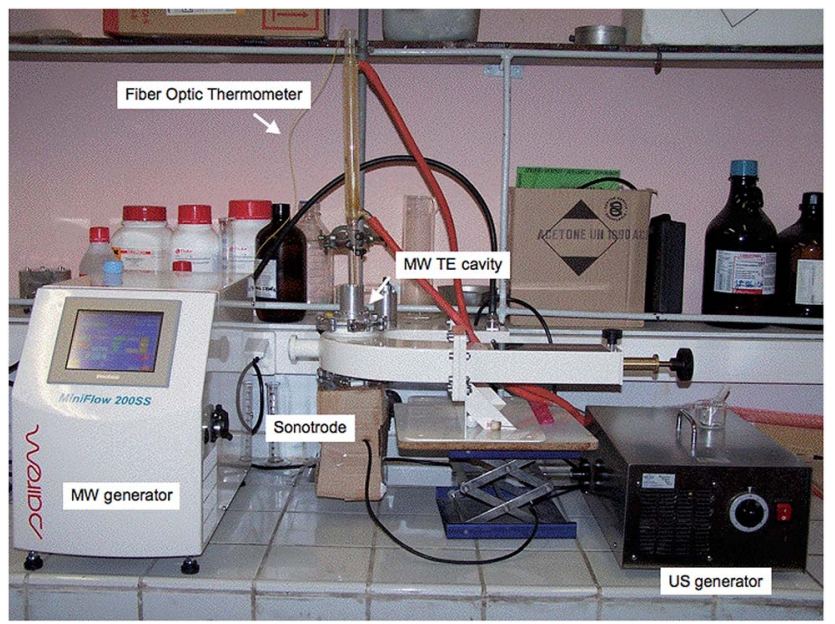

Fig. 1 Test rig: modified monomode SAIREM Miniflow 200SS cavity for simultaneous microwave/ultrasound irradiation (SMUI). semiconductor (transistor) microwave generator in the build-up of the MiniFlow, it was equally possible to optimize the $P_{\mathrm{a}}$ during the reaction by using the auto-tuning function ${ }^{58}$ which allows to decrease the level of the $P_{\mathrm{r}}$ as the reaction proceeds and the reaction mixture changes the initial parameters (reagents' concentration, temperature etc.). The new designed SMUI reactor allowed the operation without any risk of microwave leakage or damage to the built-in fibre optic temperature probe while ultrasound was on. All reactions assisted by MW, assisted by US or hybrid MW/US (SMUI) were carried out using the same pyrex reactor and the set-up described in Fig. $1 .^{59}$

Once the technical part of the newly developed system and its related safety concerns were addressed and resolved, the test rig was tested in the ring-closing metathesis reaction using glycerol micellar conditions. We previously reported the ruthenium catalyzed ring-closing metathesis (RCM) with microwave activation in non-conventional media, such as poly(ethylene glycol)s, ${ }^{36}$ glycerol, ${ }^{35}$ or in micellar conditions in water. ${ }^{60} \mathrm{We}$ also investigated the fundamental aspects of micellization in glycerol, ${ }^{28}$ determining the critical micellar concentration (CMC) of various mono and dicationic (gemini) surfactants. Based on our previous experience, the promising SMUI hybrid technology was applied to study RCM reaction in nanoreactors like micelles in glycerol. Diallyl diethyl malonate 1 (DEDAM) was selected as model substrate and its reactivity was explored with various metathesis catalysts (Scheme 1) under microwave irradiation and ultrasound, used separately or in association (Table 1).

Table 1 RCM under either MW or US, or in a combined manner (SMUI)

\begin{tabular}{llllll}
\hline & & \multicolumn{4}{c}{ Activation technique and yield $^{a}(\%)$} \\
\cline { 3 - 6 } Entry & {$[\mathrm{Ru}]$} & $\mathrm{MW}^{b, c, 61}$ & $\mathrm{US}^{b, d}$ & SMUI $^{b, e}$ & $\Delta^{f}(\%)$ \\
\hline 1 & G-I & 59 & n.p. & 58 & - \\
2 & G-II & 41 & n.p. & 62 & +21 \\
3 & HG-I & 71 & $48^{d}(9)^{g}$ & 86 & +15 \\
4 & HG-II & 38 & n.p. & 42 & +4 \\
5 & M2 & 38 & $3^{d}$ & $79^{h}$ & +41 \\
6 & M2 & 33 & n.p. & 45 & +12 \\
7 & M3 $_{1}$ & 39 & n.p. & 73 & +34 \\
8 & M5 $_{1}$ & 46 & n.p. & 66 & +20 \\
9 & M5 $_{2}$ & 13 & $39^{d}$ & 66 & +53
\end{tabular}

${ }^{a}$ Yields were calculated by ${ }^{1} \mathrm{H}$ NMR using $\mathrm{CH}_{2} \mathrm{Br}_{2}(10 \mu \mathrm{L})$ as internal standard. ${ }^{b}$ Reaction conditions: $1 \quad(0.318 \mathrm{mmol}$, substrate concentration was $0.16 \mathrm{mM}$ ), catalyst (5 mol\%), glycerol/12-2-12 mixture (2.4 g), 12-2-12 (30 mg), $1100 \mathrm{~s}(\mathrm{ca} .18 \mathrm{~min})$ at $50{ }^{\circ} \mathrm{C}$ using the specified activation technique. ${ }^{c}$ The microwave irradiation was performed by setting the MiniFlow apparatus at $P_{\mathrm{f}}=10 \mathrm{~W}$. ${ }^{d}$ Ultrasound irradiation was achieved through a metallic plate-probe fixed at the bottom of the reaction vessel (indirect sonication). ${ }^{e}$ The microwave irradiation was performed by setting the MiniFlow apparatus at $P_{\mathrm{f}}=10 \mathrm{~W}$ with integrated simultaneous ultrasound irradiation using the metallic plate-probe. ${ }^{f}$ Increase in yield observed under SMUI with respect to MW irradiation alone. ${ }^{g}$ Ultrasound irradiation was performed using a dedicated ultrasound apparatus with titanium alloy inserted into the vessel (direct sonication) the microtip temperature was $50{ }^{\circ} \mathrm{C}$, the wave amplitude was $20 \%$. ${ }^{h}$ Reaction time was $2700 \mathrm{~s} \mathrm{(45} \mathrm{min);} \mathrm{n.p.}=$ non-performed. 
We previously reported that the RCM of DEDAM 1 led to moderate yields of cyclized product 2 when the reaction was performed in pure glycerol as solvent ${ }^{35}$ (leading to the formation of glycerol ester by-products or to substrate polymerization). By performing the reaction in hydrophobic micelle core using the dicationic (gemini) 12-2-12 surfactant (CMC $=9.65 \mathrm{mM}$ in glycerol), ${ }^{28}$ the substrate was protected from the formation of glycerol esters. ${ }^{35}$

Microwave activation alone was firstly applied: the substrate DEDAM 1 was fully converted only with G-II and HG-I catalysts (Table 1, entries 2 and 3 respectively), with HG-I leading to a better yield. In all other cases, ${ }^{1} \mathrm{H}$ NMR analyses of the crude showed the presence of variable quantities of unreacted DEDAM 1 (from traces to $28 \%$ with G-I), that together with polymerization $^{28}$ and possible catalyst degradation accounted for moderate yields. The catalytic activity of second generation benzylidene G-II (entry 2) catalyst and its indenylidene counterparts $\mathbf{M} 2, \mathbf{M} 2_{0}$ and $\mathbf{M} 3_{1}$ (entries $5-7$ ) was similar. It was demonstrated that the activity of HG-II catalyst in solution can be enhanced by introduction of electron-withdrawing groups. ${ }^{62}$ Moreover, it is also generally accepted that faster initiation rate is related to lower stability of (pre)catalyst. If so, a slower initiator ${ }^{63}$ HG-II (entry 4 ) should provide higher yield at the end of the reaction than the electronically activated $M 5_{1}$ and $M 5_{2}$ (entries 8 and 9 respectively). ${ }^{64}$ This trend is not in agreement with the experimental data (Table 1), suggesting that the catalyst stability (Ru-indenylidene catalysts were more robust than the Ru-benzylidene counterparts) ${ }^{65}$ or differences in their initiation rates could not fully justify the similar (however low !) yields obtained for each group of Ru-center (indenylidene $v s$. benzylidene derivatives, or native HG-II $v s$. substituted HG-II catalysts). Indeed the Ru-coordinated electron-withdrawing ketone functionality at the isopropoxy moiety in the second generation Hoveyda-Grubbs-II type catalysts initiates faster than the parent HG-II catalyst. Other factors than (pre)catalyst stability could be more important in the (heterogeneous) system herein described. For example, mass transport, usually not significant in homogeneous catalysis, becomes fundamentally important in heterogeneous systems. Moreover the presence of ethylene, in the medium may contribute to decomposition/ deactivation of second generation Hoveyda-type catalysts. ${ }^{66,67}$ As previously demonstrated by ${ }^{1} \mathrm{H}$ NMR experiments in aqueous system, the ethylene generated in situ, was not immediately released out the micelle, probably because of the hydrophobic interactions with the saturated C-12 chains of the surfactant. ${ }^{60}$ In the case of glycerol, the higher viscosity of the medium would prevent the evaporation of the gaseous ethylene outside the micelle core, with its consequent accumulation. In fact, the formation of thick and stable foam was observed during the reaction, remaining stable for more than one hour in the absence of work-up.

To elucidate whether combination of microwaves and ultrasound could have a direct effect upon the reaction system, comparative experiments were performed setting microwave parameters as detailed in Table 1 , but simultaneously applying the ultrasound (SMUI). Ultrasound and microwave sources were switched on at the same time (200 W for US and $10 \mathrm{~W}$ MW) and the irradiation was carried out for $1100 \mathrm{~s}$ (ca. $18 \mathrm{~min}$ ). Compared to the results obtained using microwaves alone, under combined SMUI the product yield resulted 21\% higher with G-II (Table 1, entry 2), increased with HG-I (entry 3), M2 (entry 6) and $\mathbf{M}_{1}$ (entry 8) catalysts and significantly increased with $\mathbf{M} 2$ (entry 5), M3 $3_{1}$ (entry 7 ) and $\mathbf{M} 5_{2}$ (entry 9) catalysts. In this last case, the yield was increased by $53 \%$, while the SMUI technique was ineffective to raise the yields of cyclized product 2 with G-I and HG-II catalysts (Table 1, entries 1 and 4). The simultaneous use of both radiations did not allow decreasing the reaction time compared to experiments using microwave irradiation alone. However, conversion of the starting material went to completion in most cases (Table 1, entries 2-8), with the exception when using G-I (entry 1) and $\mathbf{M} 5_{2}$ (entry 9) catalysts. ${ }^{1} \mathrm{H}$ NMR analysis of the crude showed that the residual quantity of substrate 1 decreased from $28 \%$ (under MW) to $15 \%$, (under SMUI) with G-I catalyst (entry 1), and from 13\% (under MW) to $6 \%$ (under SMUI) with $\mathrm{M}_{2}$ catalyst (entry 9). Together with polymerization ${ }^{28}$ side-products, traces of isomerized compound 3 were detected by ${ }^{1} \mathrm{H}$ NMR and LC/MS analyses of the crude, only in the case of RCM reactions using G-II (entry 2) and M2 (entry 6) catalysts. Moreover, and for all catalysts, extended reaction time $(2700 \mathrm{~s})$ or more diluted conditions $(0.08 \mathrm{mM})$ were not beneficial to improve either conversions or yields.

As shown in Table 1, under SMUI irradiation, HoveydaGrubbs type $\mathrm{M5}_{1}$ (entry 8) and $\mathbf{M} 5_{2}$ (entry 9) pre-catalysts performed similarly (66\% yield of product 2 ) and were more efficient than HG-II catalyst (entry 4). This behaviour was markedly different when compared to data obtained when using microwaves. It is suggested ${ }^{68}$ that the comparable efficiency of $\mathrm{M5}_{1}$ and $\mathrm{M5}_{2}$ (pre)catalysts under SMUI come from a longer lifetime, due to a more progressive liberation of the active propagating species into the reaction medium. The stimulus provided by mechanochemical forces to dissociate the ligand, allows to release the active catalytic species, ${ }^{69}$ supporting a mechanical rather than a thermal mechanism of catalyst activation. These experimental data could be explained by taking into account the stability and the solubility of each pre-catalyst inside the micelle core, and the geometry around the ruthenium center. ${ }^{70}$

Under microwave irradiation the Ru-benzylidene catalyst G-II (Table 1, entry 2) performed similarly to the Ru-indenylidene counterparts M2 (entry 5), M2 (entry 6) and $\mathrm{M} 3_{1}$ (entry 7), while under SMUI conditions, Ru-indenylidene M2 and M3 catalysts performed much better. However, under SMUI and contrarily to expectations, the good efficiency of $\mathrm{M} 3_{1}$ (entry 7) similar to M2 (entry 5) - cannot be explained by its stability. In fact in solution, $3^{\text {rd }}$ generation catalysts (able to release the pyridine ligand) are among the fastest initiators known and their thermal stability as well as efficiency in RCM is relatively low. ${ }^{71} \mathrm{It}$ is worth noting that, under similar reaction conditions using SMUI, the indenylidene Ru-catalyst M2 (entry 5) afforded better yields compared to its Ru-benzylidene counterpart G-II (entry 2), probably because of a higher robustness.

Because the HG-I led to better yield under SMUI (entry 3), it was selected for further optimization studies: lowering the catalytic loading (2.5 mol\%) proved to be ineffective $65 \%$ yield of cyclized product 2), further increase in irradiation under 
SMUI, performed at $20 \mathrm{~W}$ or $30 \mathrm{~W}$ led to a dramatic decrease (40\% and $30 \%$ yield respectively). In order to assess the SMUI effect, the RCM reaction was investigated by irradiating the mixture by ultrasound alone. The reactivity of three different catalysts was investigated: HG-I, M2 and M5 2 (Table 1, entries 3, 5 and 9), selected on the base of their diverse chemical structures (Hoveyda-Grubbs or indenylidene types, Scheme 1) and choosing those displaying a better and/or more pronounced increased yield under SMUI compared to microwaves alone (Table 1). Thus, DEDAM 1 was sonicated at $200 \mathrm{~W}$ for $1100 \mathrm{~s}$, while keeping the temperature of the mixture constant at $50{ }^{\circ} \mathrm{C}$, as measured by the optic fiber temperature probe inside the vessel. The reaction mixture was cooled constantly with cold water flowing through an external jacket (Fig. 2 in ESI $\dagger$ ) inserted in the (microwave) cavity. As described before, the ultrasound plate-probe was located in the lower part of the microwave cavity, which was filled with water to allow the transmission of ultrasound energy to the reaction mixture (indirect sonication). DEDAM 1 was fully converted only with the indenylidene M2 (entry 5) and the Hoveyda-Grubbs type $\mathbf{M 5}_{2}$ pre-catalysts (entry 9). However, in all cases and in comparison with the data obtained under SMUI, the yields of cyclized product 2 , evaluated by ${ }^{1} \mathrm{H}$ NMR, were always lower when using ultrasound alone. This was due to a fast polymerization side reaction, ${ }^{72-74}$ but catalyst decomposition could not be excluded under the drastic ultrasound conditions. This could also account that substrate conversion was not complete when using HG-I pre-catalyst (entry 3). In fact, apart from the intrinsic stability and/or catalytic activity of complex/substrate combinations, the way by which ultrasound energy was transferred to the sample could affect the final outcome. To verify this hypothesis, the experiment was repeated with HG-I pre-catalyst by replacing the indirect plate-probe/water bath sonication with a microtip titanium probe directly immersed into the reaction vessel (direct sonication) (entry 3). The sample was irradiated for 1100 $\mathrm{s}$, setting the microtip temperature at $50{ }^{\circ} \mathrm{C}$ and the wave amplitude at $20 \%$ (Table 1 ). The cyclized product 2 was obtained in even lower yield (9\%) despite the presence of a residual quantity of substrate in the crude. Fast catalyst decomposition and products from acyclic diene metathesis (ADMET) oligomerization ${ }^{75}$ could account for this fact. In particular, reaction in the micelle core results in a 'highly concentrated' medium similar to neat conditions, already described to favour oligomerization under ultrasound. ${ }^{28,74}$ This is due to the high intensity sonication, delivering a focused energy within a small concentrated area, contrarily to the indirect sonication method with the plate-probe sonicator for which the ultrasonic intensity delivered inside the vessel was lower. In fact, the ultrasonic waves first needed to cross the liquid inside the cavity and then the wall of the reactor, before reaching the sample. To exclude that the better yield obtained with indirect sonication (plate-horn) was not due to the heat transmitted to the sample by convection, but cavitation phenomena needed to be evoked for this specific case, a comparative experiment performed in an oil-bath was performed in the same experimental conditions, i.e. $50{ }^{\circ} \mathrm{C}$ for $1100 \mathrm{~s}$. The reaction was monitored by TLC and showed full conversion of the starting material, while the cyclized product was not detected according to the ${ }^{1} \mathrm{H}$ NMR spectrum of the crude. This clearly showed not only that merely heating effect could be excluded for ultrasound-mediated (or microwave activated) experiments but also that SMUI irradiation had beneficial effect on this transformation.

For all the experiments reported in Table 1, a general workup protocol was involved. At the end of the reaction, the cyclized RCM product 2 was insoluble in the micellar media. ${ }^{60}$ It was expelled from the nanoreactor leading to the formation of two immiscible phases (glycerol/product). Consequently, the recovery of the final product 2 was achieved by decantation using a glycerol-immiscible and renewable solvent such as 2methyl-tetrahydrofuran (2-Me-THF), in which the surfactant was also insoluble. Accordingly, using the biomass derivative solvent 2-Me-THF and avoiding the use of any other toxic organic solvent maintained the environmental benefit of using glycerol as a solvent.

The data reported in Table 1 clearly demonstrated that the simultaneous use of microwaves and ultrasound was beneficial for RCM of DEDAM 1, occurring in heterogeneous media, ${ }^{60}$ affording improved yields of cyclized product 2, with diverse ruthenium catalysts (Table 1, entries 2, 3, 5, 7-9). SMUI showed the biggest positive influence on RCM reactions promoted by indenylidene Grubbs-II derivative M2 (entry 5) and HoveydaGrubbs II-type $\mathrm{M5}_{2}$ (entry 9 ) catalysts. The process benefits from rapid dielectric heating (by the presence of glycerol, ionic surfactant and solid catalyst), acoustically strengthened mass transfer and microemulsification (as solid-liquid interfaces), enhanced absorption of acoustic energy by cavitation in glycerol medium, determining a more efficient energy transfer (in viscous and heterogeneous systems) to the molecules of reactants. In heterogeneous conditions, ${ }^{\mathbf{6}}{ }^{\text {these }}$ effects become even more pronounced due to surface effects of both microwave heating (temperature effect at surface) as well as mechanical effects of ultrasound developed on the surface of the solid catalyst (microjets). The increased catalyst activity under SMUI reflected the enhanced survival probability of the free N-heterocyclic carbenes (NHCs), which might be stabilized by hydrophobic interactions with the C-12 lipophilic chains inside the micelle core, which in turn could result in higher steady state concentration of the active species. ${ }^{76,77}$ However, secondary sonochemical processes such as thermal effects leading to radical formation, could be responsible of the always observed substrate polymerization, and/or catalyst deactivation. ${ }^{76}$

\section{Conclusions}

From an economical and environmental point of view, water or 'water-like solvents' such as the biomass derivative glycerol (also defined as 'organic-water' ${ }^{27}$ ) are very attractive media for organic reactions. This approach is particularly valuable when coupled to a promising technological innovation, for which the full potential still needs to be explored, based on the irradiation of the reaction mixture by a combination of microwaves and ultrasound used simultaneously. This emerging hazard-free technique now available, allowing a real-time monitoring of 
the microwave energy adsorbed by the sample, as well as accurate control of the reaction parameters, will be profitable especially when enhanced heat transfer (provided by both MW or ultrasound irradiation) and mass transport (mainly enhanced by US) are required, in particular for heterogeneous or particularly viscous systems. As here demonstrated, this hybrid technology was successfully applied to the ring closing metathesis reaction. It is also worth noticing that the expected reactivity of Ru-complexes in glycerol micellar systems under SMUI was very often modified compared to the reactivity profiles usually observed in homogeneous conditions and in solutions. Usually carried out in non-polar organic solvents, catalysis in glycerol micellar conditions opens new perspectives and approaches for expanding the scope of glycerol use. In particular, in the case of ring closing metathesis reaction, the advantage relays on the use of commercially available catalysts without need to synthesize specific (soluble) catalysts ${ }^{78}$ or to use 'catsurf ${ }^{79,80}$ (catalysts behaving as surfactants, ${ }^{42}$ explored in aqueous micellar conditions). Further studies are in progress to extend the field of application and the full potential (and limitation) of micellar glycerol catalysis using SMUI hybrid technology.

\section{Experimental section}

\section{General experimental procedure for RCM}

12-2-12 (30 $\mathrm{mg}, 0.048 \mathrm{mmol}$ ) and the ruthenium catalyst ( $5 \mathrm{~mol} \%$ ) were gently stirred to obtain a homogenous powder. A solution of 12-2-12 in glycerol (2.4 g, prepared using $5 \mathrm{mg}$ of surfactant per $\mathrm{g}$ of glycerol), was heated gently under vigorous stirring at $60{ }^{\circ} \mathrm{C}$ for $10 \mathrm{~min}$. The solution was left to reach room temperature under stirring and added into the 12-2-12/ ruthenium solid mixture. Vigorous stirring was pursuit for additional 5 minutes, then diethyl diallylmalonate 1 (76.6 mg, $0.318 \mathrm{mmol}$ ) was added. The suspension was reacted at $50{ }^{\circ} \mathrm{C}$

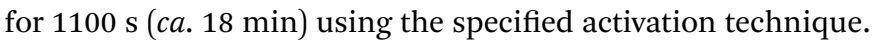
For RCM using microwave irradiation alone. The microwave irradiation was performed at $10 \mathrm{~W}$ forward power. For RCM using ultrasound irradiation alone (Indirect sonication). The ultrasound were applied through a plate-probe fixed at the bottom of the reaction vessel (sonotrode), as shown in Fig. 1. The temperature was measured by a fiber optic probe inserted into the vessel and kept constant at $50{ }^{\circ} \mathrm{C}$ using a reflux condenser (Fig. S2 in the ESI $\dagger$ ). For RCM using simultaneous microwave and ultrasound irradiation. The ultrasound and microwave sources were switched on simultaneously: the microwave irradiation was performed at $10 \mathrm{~W}$ forward power with the integrated simultaneous ultrasound irradiation using plate-probe (power level: nominal power $200 \mathrm{~W}$, transmitted power $1 \mathrm{~W} \mathrm{~mL}^{-1}$ ) and the mixture was irradiated by combined microwave and ultrasound irradiation for $1100 \mathrm{~s}$.

\section{General work-up procedure}

Upon completion of the reaction (monitored by TLC: petroleum ether-diethyl ether $7: 3 \mathrm{v} / \mathrm{v}$, iodine indicator), the mixture was cooled to room temperature. The cyclized product 2 was separated using a simple work-up: 2-methyl-tetrahydrofuran $(4 \mathrm{~mL})$ and aqueous $\mathrm{NaCl}_{\text {sat. }}(2 \mathrm{~mL})$ were added directly into the reaction vessel. The mixture was vigorously stirred for 5 min. The surfactant was not soluble in 2-Me-THF, while the cyclized product 2 was in the upper organic layer. The organic layer was decanted, dried over $\mathrm{MgSO}_{4}$, filtered, and evaporated under reduced pressure. Yields are reported in Table 1.

\section{Acknowledgements}

Martina Sacco gratefully acknowledges the help of the European Union, Erasmus Placement Program, Università de L'Aquila Italy for the mobility grant. The authors are grateful to PennAKem, Renewable Resource Chemistry, USA for providing the 2methyl-tetrahydrofuran, to Umicore AG \& Co. for the gift of M2, $\mathrm{M} 2_{0}, \mathrm{M} 3_{1}, \mathrm{M} 5_{1}, \mathrm{M} 5_{2}$ catalysts, to SAIREM, (Neyron, France) for providing both the microwave equipment Miniflow 200SS and financial support, to REUS (Contes, France) for the loan of the ultrasound equipment and to Dr Dominique Chantreux for technical support throughout the project.

\section{Notes and references}

1 F. M. Kerton, in Alternative Solvents for Green Chemistry, ed. J. Clarck, RSC Green Chemistry Series, 2009.

2 J. H. Clark and S. J. Tavener, Org. Process Res. Dev., 2006, 11, 149-155.

3 R. B. N. Baig and R. S. Varma, Chem. Soc. Rev., 2012, 41, 1559.

4 C. O. Kappe, Angew. Chem., Int. Ed., 2004, 43, 6250-6284.

5 G. Cravotto and P. Cintas, The Combined Use of Microwaves and Ultrasound: Methods and Practice, in Microwaves in Organic Synthesis, ed. A. de la Hoz and A. Loupy, WileyVCH Verlag GmbH \& Co. KGaA, Weinheim, Germany, 3rd edn, 2012, vol. 1, ch. 13, DOI: 10.1002/9783527651313.

6 G. Cravotto and P. Cintas, Chem.-Eur. J., 2007, 13, 19021909.

7 G. Cravotto, D. Garella, E. Calcio Gaudino and J.-M. Lévêque, Chem. Today, 2012, 30, 33-35.

8 A. Barge, S. Tagliapietra, L. Tei, P. Cintas and G. Cravotto, Curr. Org. Chem., 2008, 25, 1588-1612.

9 M. Draye and J. P. Bazureau, Ultrasound and Microwaves: Recent Advances in Organic Chemistry, Transworld Research Network, Kerala, India, 2012.

10 M. Maeda and H. Amemiya, New J. Chem., 1995, 19, 10231028.

11 F. Chemat, M. Poux, J.-L. Di Martino and J. Berlan, Journal of Microwave Power and Electromagnetic Energy, 1996, 31, 19-22.

12 F. Chemat, M. Poux and S. A. Galema, J. Chem. Soc., Perkin Trans. 2, 1997, 2371-2374.

13 G. Cravotto, L. Boffa, J.-M. L'evêque, J. Estager, M. Draye and W. Bonrath, Aust. J. Chem., 2007, 60, 946-950.

14 Y. Peng and G. Song, Green Chem., 2001, 3, 302-304.

15 Y. Peng and G. Song, Green Chem., 2002, 4, 349-351.

16 Y. Peng and G. Song, Green Chem., 2003, 5, 704-707.

17 Y. Peng, G. Song and R. Dou, Green Chem., 2006, 8, 573-575.

18 G. Cravotto, E. Calcio Gaudino, L. Boffa, J. M. Lévêque, J. Estager and W. Bonrath, Molecules, 2008, 13, 149-156. 
19 A. Čížová, I. Sroková, V. Sasinková and A. Malovíková, Starch/ Staerke, 2008, 60, 389-397.

20 G. Cravotto, L. Boffa, M. Bia, W. Bonrath, M. Curini and G. A. Heropoulos, Synlett, 2006, 2605-2608.

21 Y. Peng, R. Dou, G. Song and J. Jiang, Synlett, 2005, 22452247.

22 H. Feng, Y. Li, E. V. Van der Eycken, Y. Peng and G. Song, Tetrahedron Lett., 2012, 53, 1160-1162.

23 G. Cravotto, L. Orio, E. Calcio Gaudino, K. Martina, D. Tavor and A. Wolfson, ChemSusChem, 2011, 4, 1130-1134.

24 H. Feng, X. Ying, Y. Peng, E. V. Van der Eycken, C. Liu, S. Zhao and G. Song, Monatsh. Chem., 2013, 144, 681-686.

25 G. Palmisano, W. Bonrath, L. Boffa, D. Garella, A. Barge and G. Cravotto, Adv. Synth. Catal., 2007, 349, 2338-2344.

26 P. Cintas, S. Tagliapietra, E. Calcio Gaudino, G. Palmisano and G. Cravotto, Green Chem., 2014, 16, 1056-1065.

27 A. Karam, N. Villandier, M. Delample, C. K. Koerkamp, J.-P. Douliez, R. Granet, P. Krausz, J. Barrault and F. Jérôme, Chem.-Eur. J., 2008, 14, 10196-10200.

28 A. Hamel, M. Sacco, N. Mnasri, F. Lamaty, J. Martinez, F. De Angelis, E. Colacino and C. Charnay, ACS Sustainable Chem. Eng., 2014, 2, 1353-1358.

29 Handbook of Metathesis, ed. R. H. Grubbs, Wiley-VCH, Weinheim, 2003.

30 Regulation (EC) No 1907/2006 of the European Parliament and of the Council of 18 December 2006 concerning the Registration, Evaluation, Authorisation and Restriction of Chemicals (REACH), establishing a European Chemicals Agency. http://ec.europa.eu/enter.

31 K. Skowerski, J. Białecki, A. Tracza and T. K. Olszewski, Green Chem., 2014, 16, 1125-1130.

32 K. M. Kuhn, T. M. Champagne, S. H. Hong, W.-H. Wei, A. Nickel, C. W. Lee, S. C. Virgil, R. H. Grubbs and R. L. Pederson, Org. Lett., 2010, 12, 984-987.

33 X. Miao, C. Fischmeister, C. Bruneau and P. H. Dixneuf, ChemSusChem, 2008, 1, 813-816.

34 H. Bilel, N. Hamdi, F. Zagrouba, C. Fischmeister and C. Bruneau, Green Chem., 2011, 13, 1448.

35 N. Bakhrou, F. Lamaty, J. Martinez and E. Colacino, Tetrahedron Lett., 2010, 51, 3935-3937.

36 X. Bantreil, M. Sidi-Ykhlef, L. Aringhieri, E. Colacino, J. Martinez and F. Lamaty, J. Catal., 2012, 294, 113-118.

37 M. Smoleń, M. Kędziorek and K. Grela, Catal. Commun., 2014, 44, 80-84.

38 C. S. Adjiman, A. J. Clarke, G. Cooper and P. C. Taylor, Chem. Commun., 2008, 2806-2808.

39 J. Song, M. Hou, G. Liu, J. Zhang, B. Han and G. Yang, J. Phys. Chem. B, 2009, 113, 2810-2814.

40 R. Martin, C. Murruzzu, M. A. Pericas and A. Riera, J. Org. Chem., 2005, 70, 2325-2328.

41 A. Furstner, L. Ackermann, K. Beck, H. Hori, D. Koch, K. Langemann, M. Liebl, C. Six and W. Leitner, J. Am. Chem. Soc., 2001, 123, 9000-9006.

42 B. H. Lipshutz, S. Ghorai and G. T. Aguinaldo, Adv. Synth. Catal., 2008, 350, 953-956.

43 M. B. Dinger and J. C. Mol, Eur. J. Inorg. Chem., 2003, 28272833.
44 M. B. Dinger and J. C. Mol, Organometallics, 2003, 22, 10891095.

45 C. Leonelli and T. J. Mason, Chem. Eng. Process., 2010, 49, 885-900.

46 A. e. a. Canals, Patent Spain, ES2,304,839, 2009.

47 Z. Wu, E. Borretto, J. Medlock, W. Bonrath and G. Cravotto, ChemCatChem, 2014, 6, 2762-2787, and references cited therein.

48 G. Cravotto, S. Di Carlo, M. Curini, V. Tumiatti and C. Roggero, J. Chem. Technol. Biotechnol., 2007, 82, 205208, and references cited therein.

49 G. Cravotto, G. Omiccioli, C. Buffa and G. Dal Lago, VR 2004A000167, 2004.

50 V. Ragaini, C. Pirola, S. Borrelli, C. Ferrari and I. Longo, Ultrason. Sonochem., 2012, 19, 872-876.

51 I. Longo and V. Ragaini, WO/2007/093883, 2007.

52 A. Hernoux, J.-M. Lévêque, U. Lassi, S. Molina-Boisseau and M.-F. Marais, Carbohydr. Polym., 2013, 92, 2065-2074.

53 C. Domini, L. Vidal, G. Cravotto and A. Canals, Ultrason. Sonochem., 2009, 16, 564-569.

54 C. Buffa, G. Cravotto and G. Omiccioli, TO A-000766, 2006.

55 X.-F. Shen, J. Chem. Technol. Biotechnol., 2009, 84, 18111817.

56 S. Chemat, A. Lagha, H. A. Amar and F. Chemat, Ultrason. Sonochem., 2004, 11, 5-8.

57 Y. Coquerel, E. Colacino, J. Rodriguez, J. Martinez and F. Lamaty, Microwave-Assisted Stereoselective Synthesis, in Stereoselective Synthesis of Drugs and Natural Products, ed. V. Andrushko and N. Andrushko, Wiley, 2013, vol. 1, ch. 5, online ISBN: 9781118596784 , DOI: 10.1002/9781118596784.

58 L. Latrasse, M. Radoiu, J.-M. Jacomino and A. Grandemenge, Facility for microwave treatment of a load, WO 2012146870, 2012.

59 The SMUI hybrid technology can also be scaled-up. Direct sonication is achieved in dedicated apparatus, equipped with a non-metallic horn inserted in SAIREM multimodal microwave cavity. More data and technical details can be provided by SAIREM (Neyron, France).

60 L. Laville, C. Charnay, F. Lamaty, J. Martinez and E. Colacino, Chem.-Eur. J., 2012, 18, 760-764.

61 The comparative study of the energy efficiency in RCM process performed with the MiniFlow 200SS and other brands of microwave assisted reactors is not a trivial challenge. The experimental results reported in Table 1 can not be compared to those ones described by us in ACS Sustain. Chem. Eng. 2014, 2, 1358-1358 (ref. 28), whose the real energy absorbed can not be measured but only on the temperature measured at one or multiple points inside the reactor.

62 A. Michrowska, L. Gulajski, Z. Kaczmarska, K. Mennecke, A. Kirschning and K. Grela, Green Chem., 2006, 8, 685-688.

63 S. Guidone, E. Blondiaux, C. Samojłowicz, Ł. Gułajski, M. Kędziorek, M. Malińska, A. Pazio, K. Woźniak, K. Grela, A. Doppiu and C. S. J. Cazin, Adv. Synth. Catal., 2012, 354, 2734-2742.

64 V. Thiel, K.-J. Wannowius, C. Wolff, C. M. Thiele and H. Plenio, Chem.-Eur. J., 2013, 19, 16403-16414. 
65 Y. Schrodi and R. L. Pederson, Aldrichimica Acta, 2007, 40, 45-52.

66 S. H. Hong, A. G. Wenzel, T. T. Salguero, M. W. Day and R. H. Grubbs, J. Am. Chem. Soc., 2007, 129, 7961-7968.

67 J. A. M. Lummiss, N. J. Beach, J. C. Smith and D. E. Fogg, Catal. Sci. Technol., 2012, 2, 1630-1632.

68 M. Bieniek, C. Samojlowicz, V. Sashuk, R. Bujok, P. Sledz, N. Lugan, G. Lavigne, D. Arlt and K. Grela, Organometallics, 2011, 30, 4144-4158.

69 A. Piermattei, S. Karthikeyan and R. P. Sijbesma, Nat. Chem., 2009, 1, 133-137.

70 S. Guidone, E. Blondiaux, C. Samojłowicz, Ł. Gułajski, M. Kedziorek, M. Malinska, A. Pazio, K. Wozniak, K. Grela, A. Doppiu and C. S. J. Cazin, Adv. Synth. Catal., 2012, 354, 2734-2742.

71 C. A. Urbina-Blanco, A. Leitgeb, C. Slugovc, X. Bantreil, H. Clavier, A. M. Z. Slawin and S. P. Nolan, Chem.-Eur. J., 2011, 17, 5045-5053.

72 C. O. Akintayo, H. Mutlu, M. Kempf, M. Wilhelm and M. A. R. Meier, Macromol. Chem. Phys., 2011, 213, 87-96.
73 P. B. Cardoso, A. Musyanovych, K. Landfester, C. Sayer, P. H. H. Araujo and M. A. R. Meier, J. Polym. Sci., Part A: Polym. Chem., 2014, 52, 1300-1305.

74 L. Gulajski, P. Sledz, A. Lupa and K. Grela, Green Chem., 2008, 10, 271-274.

75 J. C. Conrad, M. D. Eelman, J. A. Duarte Silva, S. Monfette, H. H. Parnas, J. L. Snelgrove and D. E. Fogg, J. Am. Chem. Soc., 2007, 129, 1024-1025.

76 R. Groote, R. T. M. Jakobs and R. P. Sijbesma, ACS Macro Lett., 2012, 1, 1012-1015.

77 The release of NHC ligands from palladium-based PEPPSItype complexes (PEPPSI standing for Pyridine Enhanced Precatalyst Preparation Stabilization and Initiation) under ultrasound activation, was previously demonstrated by $\mathrm{X}$ Ray Photoelectron Spectroscopy (XPS). See: A. Azua, J. A. Mata, P. Heymes, E. Peris, F. Lamaty, J. Martinez and E. Colacino, Adv. Synth. Catal., 2013, 355, 1107-1116.

78 J. P. Jordan and R. H. Grubbs, Angew. Chem., Int. Ed., 2007, 46, 5152-5155.

79 R. Gawin, P. Czarnecka and K. Grela, Tetrahedron, 2010, 66, 1051-1056.

80 B. H. Lipshutz and S. Ghorai, Org. Lett., 2009, 11, 705-709. 